\title{
REVISÃO DA DISTRIBUIÇÃO DOS GENEROS PARACALANUS, CLAUSOCALANUS E CTENOCALANUS (COPEPODA, CRUSTACEA) AO LARGO DO BRASIL
}

\author{
TAGEA K. S. BJORNBERG
}

Departamento de Zoologia, Instituto de Biociências, Universidade de Sశ̋o Paulo, SP, Brasil

\section{SYNOPSIS}

Samples cited in the papers of Björnberg $(1963,1965)$ and some taken recently off Santos, Rio de Janeiro and Cabo Frio (Brazill) were restudied. The distribution of the several species of the genus Paracalanus (crassirostris, nanus, quasimodo, parvus s. str., indicus, aculeatus), of the genus Clausocalanus (furcatus, mastigophorus, pergens, ingens, paululus, parapergens and arcuicornis) were surveyed in Brazilian waters. Two new species Paracalanus campaneri and Delius sewelli were described. Two Ctenocalanus were found in Brazilian waters not corresponding to the description of Ctenocalanus citer. The distribution of these especies was compared off South America.

\section{Introduçẵo}

Os Clausocalanus e os Paracalanus são em geral os mais numerosos primeiros consumidores pertencentes ao plâncton, em todos os oceanos, exceção das águas antárticas e de algumas águas mais frias costeiras, onde são substituídos pelos Ctenocalanus. Desde a publicação do trabalho de Björnberg (1963) sobre a distribuiçăo das espécies destes gêneros ao largo do Brasil, foram criadas algumas novas espécies e separados em gêneros novos algumas das espécies existentes. Assim, Frost \& Fleminger (1968) reestudaram os Clausocalanus e subdividiram algumas espécies, acrescentando cinco novas às já existentes e reestabelecendo uma. anteriormente colocada em sinonímia; Heron \& Bowman (1971) separaram de Ctenocalanus vanus Giesbrecht. 1888, a espécie Ctenocalanus citer; Bowman (1971) dividiu Paracalanus parvus (Claus, 1863) em três espécies: $P$. parvus sensu stricto, $P$. quasimodo Bowman, 1971 e $P$. indicus (Wolfenden, 1905); Andronov (1972) criou o novo genero Delius para abrigar Paracalanus nudus Sewell, 1929, e, descreveu do Atlântico Leste a espécie $P$. tropicus parecida com $P$. indicus e $P$. quasimodo, além de colocar em novo gênero, Parvocalanus, a conhecida espécie Paracalanus crassirostris F. Dahl, 1894 (Andronov, 1970, 1977).

Baseados na abundância destes pequenos Calanoida, tornou-se imperativa a revisão dos estudos feitos em 1963, também porque, sendo o primeiro trabalho sobre uma distribuição geral dos copépodos ao largo do Brasil, poderia conter identificaçð̄es errôneas de espécies e outras imnerfeiç̃es.

\section{Material Usado}

Constou de algumas amostras citadas nos trabalhos de Björnberg (1963 e 1965): M-37, M-98, M-242, M-244, M-245, M-497 tiradas da Corrente do Brasil, de água Tropical; M-161, M-162, M-164, M-401, M-402, M-407, M-409 de água Subtropical de Superfície; M-78 colhida entre 54 e $103 \mathrm{~m}$ em águas sobre o fundo da plataforma continental ao largo do Sul do Brasil; E-70, E-80, M-107 e IV-320 de águas costeiras; amostras da coleção Lomonosov, tiradas de profundidades entre 0 e $1040 \mathrm{~m}$ em águas Tropical, Subtropical, Central do Atlântico Sul e Intermediária Antártica. Também foram usadas amostras recentemente coletadas ao largo de Cabo Frio, santos e rio de Janeiro na superfície e até profundidades de $300 \mathrm{~m}$, as quais fazem parte do acervo de amostras de plâncton do Instituto Oceanográfico e cujos Paracalanus foram identificados pelo Dr. Antonio Frederico Campaner, a quem sou muito grata nāo só pelas informaçðes cediđas, como ainda, pela discussāo profícuadeste trabalho.

\section{Kesultados}

1) Família Paracalanidae - $P$. crassirostris F. Dahl, 1894, caracteriza as águas interiores e costeiras pela abundância e frequência; $P$. nanus G. O. Sars, 1907 , foi numeroso em amostra de água Subtropical de Superfície ao largo do Sul do Brasil; $P$. parvus Claus, 1863, sensu stricto, (vide Bowman, 1971) apareceu em amostras de águas costeiras e de plataforma ao largo de Santos; $P$. quasimodo Bowman, 1971 é o representante do gênero Paracalanus mais numeroso e abundante em águas costeiras de salinidade acima de $30 \%$, de plataforma quente e Tropical; $P$. indicus Wolfenden, 1905. menos frequente e abundante que o precedente; Paracalanus campaneri sp. nov. é um dos Paracalanus mais frequentes em amostras costeiras, de plataforma e de água Subtropical de superfície, podendo estar presente em amostras de água Tropical; do gênero Delius Andronov, 1972, registramos a presença de uma espécie nova para a ciência, $D$. sewelli, de água Subtropical de Superfície e de águas oceânicas quentes, dominante numericamente nas amostras em que foi encontrada, $P$. aculeatus ocorreu ao largo do Sul do Brasil.
2) Família Pseudocalanidae: Clausocalanus furcatus (Brady, 1883) caracteriza, pela abundância e frequência, a água Tropical da Corrente do Brasil e as águas de plataforma quentes superficiais, podendo ser abundante em águas costeiras quentes de alta salinidade e em água de plataforma de fundo, onde é frequente, e esporadicamente em água Subtropical de superfície do fim da Corrente do Brasil e da Convergência Subtropical; Clausocalanus mastigopho. rus (Claus, 1863) é frequente em água Tropical e pouco frequente em água de fundo sobre a plataforma continental; $C$. pergens Farran, 1926 é numeroso à noite em água Subtropical de superfície; $C$. ingens Frost \& Fleminger, 1968, é pouco numeroso nas mesmas águas à noite; $C$. paululus Farran, 1926 é abundante em água de plataforma de fundo e água Subtropical ao largo da costa leste do Brasi e em água Central subsuperficial do Atlántico, sendo pouco abundante de dia e na superfície destas águas; $C$. parapergens Frost \& Flaminger. 1968, è pouco numeroso em ámua Subtropical de superfície de dia e em água de fundo sobre a plataforma continental ao largo do sul do Brasil; C. arcuicornis (Dana, 1849) ocorreu sempre em pequena abundância mas, frequentemente em água de fundo sobre a plataforma continental ao Sul do Brasil e em água Subtropical de superfície; Ctenocalanus citer Heron \& Bowman, 1971, não foi registrado com certeza em nenhuma das amostras estudadas, parecendo ser característico de águas antárticas; os outros Ctenocalanus registrados em amostras tiradas ao largo de Santos e do Rio Grande do Sul, diferem uns dos outros e suas características são descritas mais adiante.

Paracalanus campaneri sp, nov. - Sinonímia: Paracalanus aculeatus Björnberg, 1963 (part.). Diagnose: $\uparrow=1,05$ a $1,03 \mathrm{~mm}$ de comprmento, o comprimento do urosoma é mais ou menos $1 / 3$ a $1 / 3$ e meio do comprimento do cefalotorax, as antênulas ultrapassam os ramos caudais em dois artículos, receptáculo seminal bifurcado em vista tateral, segundo artículo do exópodo da segunda e terceira patas natatórias espinulado, espínulos marginais externos do último artículo dos exópodos fortes e numerosos nas segundas. terceiras e quartas patas, quinto par de patas com dois ou três artículos e dois espínulos terminais e uma cérdula.

Discussão: Bradford (1978) refere-se a dois grupos no gênero Paracalanus sensu stricto: o grupo aculeatus que conteria as espécies $P$. aculeatus Giesbrecht, 1888 e $P$. denudatus Sewell, 1929; e o grupo parvus contendo $P$. parvus, $P$. nanus, $P$, indicus, $P$. quasimodo e $P$. tropicus. Vervoort (1963) coloca na sinonímia de $P$. denudatus Sewell o Paracalanus pygmeeus figurado por Sars (1925) e considerado não identificável a partir da descrição original de Claus (1863). O P. denudatus de Sewell é uma forma costeira pequena $(0,64 \mathrm{~mm})$ e é provavelmente a mesma espécie figurada por Vervoort (1963) ao largo da Åfrica no Atlântico. Possui pouca espinulação nas margens externas dos últimos artículos dos exópodos das segundas, terceiras e quartas patas, antênulas longas, ultrapassando os ramos caudais em comprimento. O P. pygmaeus de Sars mediu $0,76 \mathrm{~mm}$, segundo Vervoort (1963), mas, difere dos exemplares aqui descritos pelo tamanho menor das antênulas, pela espinulação mais abundante das margens externas dos terceiros artículos dos exópodos das patas $2,3,4$, e pela falta de espinulação na face dos segundos artículos dos exópodos das mesmas patas. Somos pela manutenção da validez desta espécie. Teríamos então no grupo aculeatus: $P$. pygmaeus Sars, $1925, P$. aculeatus Giesbrecht, 1888 , $P$. demudatus Sewell, 1929 e $P$. campaneri sp. nov. A principal diferença entre $P$. campaneri e $P$. aculeatus, espécie com a qual se parece muito, está na forma do receptáculo seminal, que em aculeatus não é bifurcado em vista lateral. Como a bifurcação é frequentemente recoberta pelas margens posteriores do torax, cremos que boa parte das identificacões de $P$. aculeatus, pelo menos a maioria identificada por nós (Björnberg, 1963), foi errônea. $P$. pygmaeus, $P$. denudatus, e $P$. campaneri tèm o receptáculo seminal bifurcado terminalmente em vista lateral. $\hat{E}$ frequente no grupo, a presença de cerdas nas margens torácicas posteriores laterais. 
Holótipo: No Museu de copépodos do Departamento de Zoologia, Instituto de Biociências, Universidade de São Paulo com n ? 184. de amostra coletada ao largo de Santos, Estado de São Paulo, Brasil; Parátipos, na mesma coleção, sob nọ 185 , da amostra M-164, coletada ao largo do Rio Grande do Sul, Brasil, em águas limítrofes com as do Uruguay.

A denominação campaneri foi dada em honra do Dr. Antonio Frederico Campaner que, além de chamar nossa atenção sobre a ocorrência desta espécie. desenhou-a para esta publicação

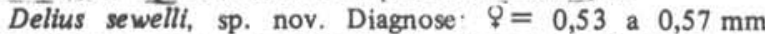
de comprimento, cabeça anteriormente achatada, espinulação das patas 1 a 4 conforme Figuras 26 a 29; 5a. pata única com 3 artículos, sendo o último muito longo, duas vezes mais comprido que o médio: $\delta=0,43 \mathrm{~mm}$, com a pata 5 possuindo artículos primeiro, terceiro e quarto aproximadamente duas vezes mais longos que o segundo e o último. Nos demais caracteres, muito parecido com o macho de D. nudus (Sewell, 1929), figurado por Andronov (1972).

Holótipo: 9 depositado no Museu de copépodos do Dep. de Zoologia, Inst. Biociências, U.S.P., sob n? 186, proveniente da amostra M-407 coletada ao largo do Rio Grande do Sul, em águas oceânicas, da Convergência Subtropical; alótipo ớ. depositado no mesmo Museu sob no 187 , com a mesma proveniência.
O nome sewell tol dado em nomenagem a R.B.Seymour Sewell. o primeiro a descrever uma espécie deste gênero.

Discussão: Dellius sewelli é menor que $D$. nudus, a cabeça é mais achatada anteriormente, as patas têm mais espínulos nas faces dos artículos e a pata 5 é muito diferente. Nos outros carateres. principalmente na forma da genitalia, as duas espécies se parecem bastante.

C'tenocalanus vanus Giesbrecht, 1888 sensu lato. é representado em águas brasileiras por duas formas; uma de antênulas curtas. a fêmea de comprimento maior $(1,35 \mathrm{~mm})$, com receptáculo seminal representado na Fig. 16, a outra, com antênulas mais longas. a fêmea de comprimento menor, (entre 1,16 e $1.26 \mathrm{~mm}$ ) com o receptáculo seminal de aspecto ligeiramente diferente (Fig. 17). Após compara ção destas formas com a originalmente descrita por Giesbrecht, poderemos concluir sobre o status taxonômico a ser conferido a cada uma.

Comparação da distribuiçāo dos gêneros ao largo da América do Sul

Ao largo do Brasil e no Mar Caribe a espécie mass abundante de Clausocalanus é $C$ furcatus, típica de águas quentes e muito salinas. Ao largo da Argentina, Ramírez (1969) assinalou ( brevipes

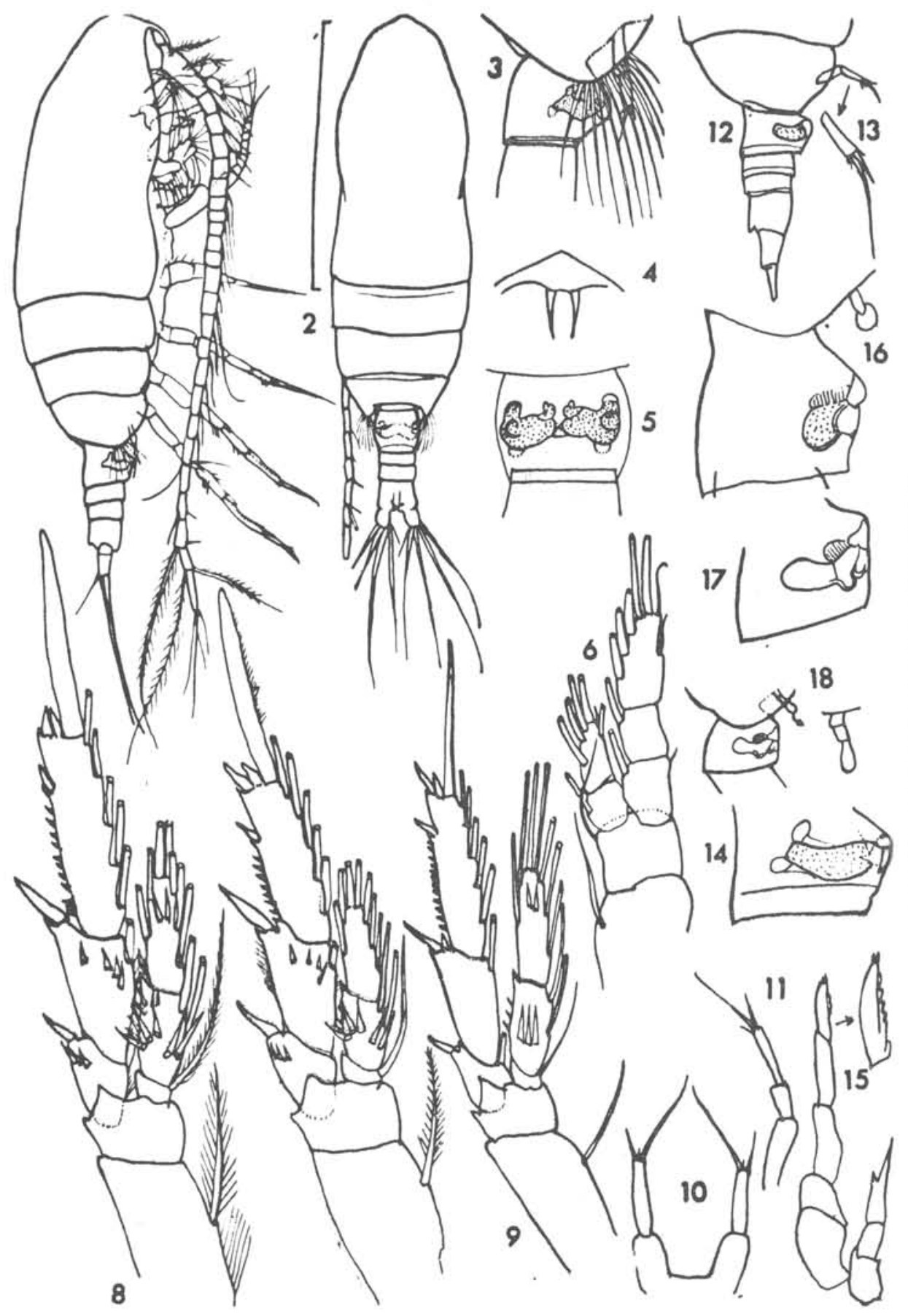

Paracalanus campaneri sp. n. - 1-Vista lateral da fêmea (escala $-0,5 \mathrm{~mm}) ; 2$ Fêmea vista dorsal; 3 - Aspecto da margem posterior do tórax e segmento genital; 4 - Rostro: 5 - Segmento genital, vista ventral; 6 a 10 . 1a a 5 a patas; 11 - 5 ạ pata de outro exemplar.

Paracalanus aculeatus Giesbrecht, $1888-12$ - Vista lateral do corpo posterior de uma fêmea; 13 - 5 a pata feminina, último artí. culo; 14 - Segmento genital, lateral; 15 . 5 aș patas de outro exemplar macho e detalhe de um dos últimos artículos.

Ctenocalanus spp. - 16 - Segmento genital e 5 a pata de uma fêmea coletada ao largo do Rio Grande do Sul, em 1,35mm de comprimento total do corpo: 17 - Segmento genital de fêmea coletada ao largo de Santos, com comprimento total do corpo $1,26 \mathrm{~mm}$, 18 - 5a pata e segmento genital desta fêmea. 


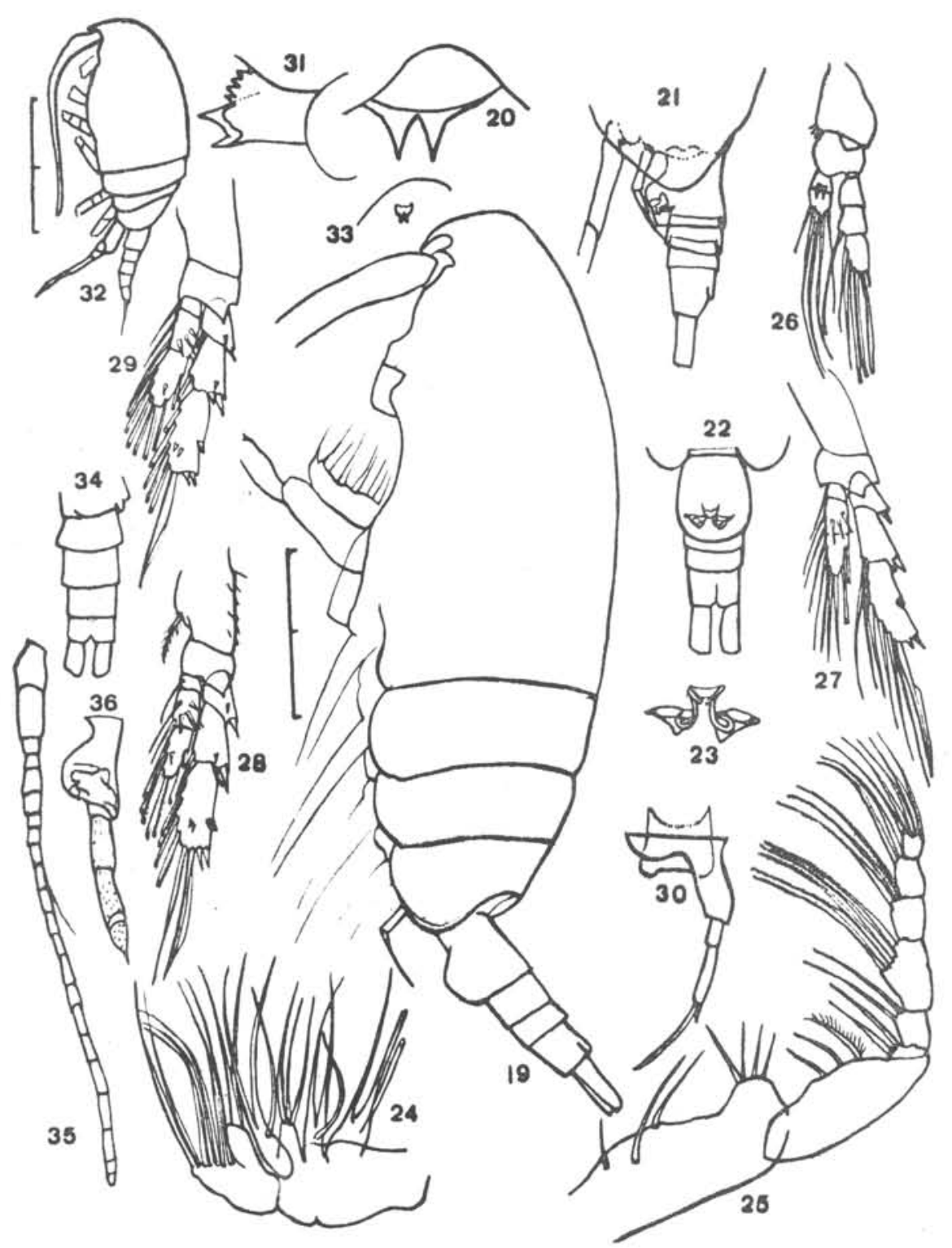

Delius sewelli sp. n. - 19 - Vista lateral de uma fêmea; 20 - Rostro da mesma;21 - Urosoma e margem posterior do tórax da fêmea; 22 - Aspecto ventral do mesmo urusoma; 23 - Genitalia feminina em detalhe; 24 - Maxila; 25 - Maxilípede; 26 a $30-1$ a a 5 a patas da fêmea; 31 - Gnatobase da mandíbula; 32 - macho lateralmente; 33 - Rostro do macho; 34 - urosoma do macho; 35 - Antênula do mesmo; 36 - 5 a pata, vista ventral. Escalas: Fig. $32=0,2 \mathrm{~mm}$; Fig. $19=0,1 \mathrm{~mm}$.

como a espécie mais abundante, característica de águas mais frias e C. laticeps como caraterística de águas da Corrente das Malvinas ou Falklands. Ao largo da costa ocidental da América do Sul, $C$. furcatus é a espécie mais numerosa em águas de superfície aquecidas, tanto costeiras como Tropical, Equatorial, e Subtropical de superfície ao largo da Colombia, e até em águas subantárticas aquecidas ao Norte do Chile. Daí para o sul, diminuı em número. É espécie de superfície. C. parapergens foi a espécie mais frequente e numerosa ao largo do Chile em águas Subantárticas, tem migração vertical para a superfície à noite e de dia vive na camada subsuperficial. $C$. arcuicornis, bem menos numeroso, foi mais abundante em águas de baixa salinidade ao largo do Chile, e não tem migração vertical. C. ingens, assinalado por Ramírez (1969) ao largo da Argentina, ocorre também no Pacífico Leste de $31^{\circ} \mathrm{S}$ a $47^{\circ} \mathrm{S}$, frequente mas pouco numeroso. $C$. laticeps foi encontrado em número elevado ao largo do Sul do Chile em águas Subantárticas e de profundidade. $C$. jobei ocorre em águas Subantárticas de superficie ao largo do norte do Chile e em água do Perú de superfície foi o mais numeroso em duas amostras examinadas. $C$. mastigophorus ocorreu em águas quentes Equatorial de superfície, Perú de superfície, Subantártica superficial e foi registrada com frequência ao largo do Norte do Chile por Vidal (1968). Ctenocaianus vanus parece ser espécie que prefere águas ricas em dinoflagelados, como as brasileiras, pois, foi raramente encontrada ao largo do Chile (Bjormberg, 1973). P. indicus foi mais numeroso no Pacífico Leste que $P$. quasimodo, ao largo do Brasil e da Argentina.

\section{Bibliografia}

ANDRONOV, V.N. 1970. Some problems of taxonomy of the family Paracalanidae (Copepoda). Zool. Zh., 49: 980-925 (Em russo).

poda, Paracalanidae). Zool. Zh., 51: $597-599$ (Em russo). - 1977 Paracalanus tropicus sv. n. (Copepoda, Paracalanidae) from South-east Atlantic. Zool. Zh., 56: 154-156 (Em russo).

BJORNBERG, T.K.S. - 1963 - On the marine roe-living copepods off Brasil. Bolm, Inst. oceanogr., S. Paulo, 13: $13-142$.

- 1965. The study of copepods in the South Atlantic waters. Anais Acad. brasial. Ciênc., 37 (Supl.): 219-230 - 1973. The planktonic copepods of the Marchile 1 expedition and of the "Elatnin" Cruises 3-6 taken in the South East Pacific. Bolm Zool. e Biol. Mar., N.S., 30: 245-394.

BOWMAN, T.E. 1971. The distribution of calanoid copepods off the South eastern United States between Cape Hatteras and Southern Florida. Smithsonian Contr. Zool., 96: 1-58.

BRADFORD, J.M. 1978. Paracalanus indicus Wolfenden and Corycaeus aucklandicus Kraemer, two neritic pelagic copepods from New Zeland. J. R. Soc. N. Z., 8: 133-141.

FROST, B. \& FLEMINGER, A. 1968. A revision of the genus Clausocalanus (Copepoda, Calanoida) with remarks on 
distributional patterns in diagnostic characters. Bull. Scripps Inst. Oceanogr., 12:1-235.

HERON, G.A. \& BOWMAN, T.E. 1971. Postnaupliar developmental stages of the copepod crustaceans Clausocalanus laticeps, C. brevipes, and Ctenocalanus citer (Calanoida, Pseudocalanidae). Antarctic Res. Ser., 17:141-165.

RAMIREZ. F.C. 1969. Copépodos planctonicos del sector Bonaerense del Atlantico Suroccidental. Datos y resultados de las campañas Pesqueria. Contr. Inst. Biol. mar., Mar del Plata, 98:1-166.
SARS, G.O. 1924-1925. Copépodes particulièrement bathypelagiques provenant des campagnes scientifiques du Prince Albert I de Monaco. Rés. Camp. Sci. Monaco, 69:1-108 (1925); Atlas (1924), 127 pls.

VERVOORT, V. 1963. Pelagic Copepoda, Part 1: Copepoda Calanoida of the families Calanidae up to and including Euchaetidae. Atantide Rep., 7: 77-194.

VIDAL, J. 1968. Copepodos calanoideos epipelágicos de la expedición MarChile II. Gayana, Zool.. 15: 1-98. 